\title{
A Phase I Dose Escalation Trial of Gemcitabine with Radiotherapy for Breast Cancer in the Treatment of Unresectable Chest Wall Recurrences
}

\author{
W. Warren Suh, MD, MPH, ${ }^{*}$ Anne F. Schott, MD, ${ }^{\dagger}$ James A. Hayman, MD, MBA,* \\ Matthew J. Schipper, MS, ${ }^{\ddagger}$ Donna S. Shewach, PhD,${ }^{\mathbb{S}}$ and Lori J. Pierce, MD* \\ Departments of *Radiation Oncology, ${ }^{\dagger}$ Internal Medicine, and ${ }^{\$}$ Pharmacology, University of Michigan, \\ and ${ }^{\ddagger}$ Biostatistics Core, University of Michigan Cancer Center, Ann Arbor, Michigan
}

\begin{abstract}
The purpose of this study was to determine the maximum tolerated dose (MTD) of gemcitabine when given concurrently with standard radiotherapy for the treatment of chest wall recurrences, and to compare actuarial rates of local-regional control with those achieved in historical controls. Patients with unresectable chest wall recurrences were enrolled in a phase I trial of concurrent gemcitabine and radiotherapy. Gemcitabine was increased at $150 \mathrm{mg} / \mathrm{m}^{2} /$ week increments, starting at $300 \mathrm{mg} / \mathrm{m}^{2} /$ week. Radiotherapy was delivered to the chest wall and regional nodes to a total of 60 to 70 Gy in 2 Gy daily fractions. Treatment toxicity was assessed and a comparison of treatment outcome was performed between study patients and historical groups treated with either radiotherapy alone or excision followed by radiotherapy. The dose-limiting toxicities of neutropenia and thrombocytopenia occurred at the second planned dose of $450 \mathrm{mg} / \mathrm{m}^{2} /$ week after accrual of only six patients, resulting in a MTD of $300 \mathrm{mg} / \mathrm{m}^{2}$ / week. Myelosuppression and skin desquamation were commonly observed. Actuarial rates of local-regional control were $100 \%$, $50 \%$, and $90 \%$ at 2 years for the gemcitabine with radiotherapy, radiotherapy alone, and excision followed by radiotherapy groups, respectively ( $p=0.105)$. The difference among the Kaplan-Meier curves for overall local-regional control was statistically significant at $p=0.007$ in favor of combined gemcitabine and radiotherapy. The MTD of gemcitabine is $300 \mathrm{mg} / \mathrm{m}^{2} / \mathrm{week}$ when gemcitabine is delivered concurrently with radiotherapy for unresectable chest wall failures. This novel approach suggests excellent localregional control when compared to historical controls. A phase II trial is warranted. .
\end{abstract}

Key Words: breast cancer, chest wall failure, gemcitabine, mastectomy, radiotherapy

A chest wall recurrence of breast cancer is defined as the appearance of tumor in the skin, subcutaneous tissue, or muscles of the chest wall following mastectomy. It may be associated with nodal failures involving the supraclavicular, axillary, and/or rarely the internal mammary regions. The incidence of an isolated breast cancer chest wall recurrence with or without regional nodal involvement after mastectomy has been reported to be from $5 \%$ to $30 \%$ in several series, largely dependent upon the extent of nodal involvement and the size of the primary tumor (1-3).

The standard treatment for an isolated local or localregional failure after mastectomy is radiotherapy, with a preceding surgical resection when feasible. In general, for patients with smaller lesions that are amenable to a complete resection, all gross disease is excised prior to proceeding with local-regional radiotherapy. These patients have an increased probability of local control, and in most

Address correspondence and reprint requests to: Lori J. Pierce, MD, UH B2C490, Box 0010, 1500 East Medical Center Dr., Ann Arbor, MI 48109-0010, USA, or e-mail: ljpierce@umich.edu.

(C) 2004 Blackwell Publishing, Inc., 1075-122X/04/\$15.00/0

The Breast Journal, Volume 10, Number 3, 2004 204-210 cases survival, when compared to those with larger unresectable lesions (4-8).

Current treatment for patients with unresectable chest wall recurrences, however, is mainly restricted to radiotherapy alone. Several centers have reported 5 -year rates of local failure as high as 66 to $76 \%$ in patients treated with full-dose radiotherapy alone versus 43 to $52 \%$ observed in those treated with excision followed by radiotherapy. Unfortunately local control at times is not durable even for those patients with surgically resectable recurrences $(4,6)$, and uncontrolled local-regional disease can adversely affect quality of life (9). Thus sustained palliation remains an important therapeutic goal, and a chest wall recurrence of breast cancer represents an excellent clinical model to study radiosensitizers in an attempt to improve localregional control.

Gemcitabine $\left(2^{\prime}, 2^{\prime}\right.$-difluorodeoxycytidine $\left.[\mathrm{dFdC}]\right)$ is a deoxycytidine analogue that elicits cancer cell death through its incorporation into DNA and inhibition of deoxynucleotide synthesis. Antitumor activity of gemcitabine has been tested in culture on multiple cell lines, and drug efficacy has been observed in human xenograft studies involving 
breast, lung, head and neck, colon, and ovarian cancers (10). Recent phase II trials have been conducted successfully in a variety of solid tumors, including breast cancer (11). In addition, radiation-sensitization properties of gemcitabine have been recently demonstrated at noncytotoxic drug levels in several solid tumor cell lines in vitro, including the human breast cancer cell line MCF-7 (12-14). Therefore, with both proven cytotoxic and radiation-sensitizing activities in breast cancer, gemcitabine represents an excellent candidate drug for radiationsensitizing studies in the treatment of unresectable chest wall failures that have been historically resistant to radiotherapy alone.

The primary objective of this phase I trial was to determine the maximum tolerated dose (MTD) of oncea-week gemcitabine and its dose-limiting toxicity (DLT) when administered concurrently with standard radiation in patients with unresectable chest wall recurrences. A secondary objective was to assess and compare the actuarial rates of local-regional control achieved with gemcitabine and radiotherapy with the rates observed in historical control patients treated at our institution.

\section{MATERIALS AND METHODS}

\section{Eligibility}

After obtaining Institutional Review Board approval, all patients with unresectable local-regional recurrence of breast cancer following mastectomy who signed informed consent forms were eligible to participate in this phase I trial. Histopathologic confirmation of a chest wall recurrence with at least $1 \mathrm{~cm}$ of gross unresectable disease with or without nodal involvement was required. Patients with synchronous distant metastases were eligible as long as their life expectancy was at least 4 months. No concurrent chemotherapy other than gemcitabine was allowed, with a mandatory washout time of at least 4 weeks from any previous chemotherapy or hormonal therapy. Additional eligibility criteria included age $\geq 18$ years, Karnofsky performance score of 60 or greater, freedom from any medical comorbidity that would limit survival to less than 2 years, and adequate organ function as demonstrated by a white blood cell count greater than $3000 / \mathrm{mm}^{3}$, absolute neutrophil count $\geq 1500 / \mathrm{mm}^{3}$, platelet count $\geq 100,000 /$ $\mathrm{mm}^{3}$, hemoglobin $\geq 10 \mu \mathrm{g} / \mathrm{dl}$, creatinine less than $2.0 \mathrm{mg} /$ $\mathrm{dl}$, aspartate aminotransferase (AST) and alanine aminotransferase (ALT) less than three times the upper limits of normal, total bilirubin less than $2.0 \mathrm{mg} / \mathrm{dl}$, prothrombin time (PT) less than 14, and partial thromboplastin time (PTT) less than 40.
Exclusion criteria consisted of any regional node involvement in the absence of chest wall failure and a history of any prior irradiation to the chest wall and/or supraclavicular fossa regions.

\section{Study Design}

Dose escalation of gemcitabine was performed while delivering a standard dose of radiotherapy. A starting dose level of $300 \mathrm{mg} / \mathrm{m}^{2} /$ week (approximately $25 \%$ of the MTD of gemcitabine when used alone) was chosen for the study. Gemcitabine was administered via once-a-week infusion at a rate of $10 \mathrm{mg} / \mathrm{m}^{2} / \mathrm{min}$. This rate was based on clinical and in vitro studies demonstrating that the accumulation rate of difluorodeoxycytidine triphosphate (dFdCTP) in mononuclear and leukemia cells was saturated when plasma dFdC levels reached 15 to $20 \mu \mathrm{mol} / \mathrm{L}$. Infusion of this drug at a rate of $10 \mathrm{mg} / \mathrm{m}^{2} / \mathrm{min}$ produced steady-state plasma levels at this range, making this schedule attractive for radiosensitizing studies $(15,16)$. Preclinical data and recent clinical trials demonstrated that gemcitabine could radiosensitize tumor cells at concentrations lower than $1 \mu \mathrm{M}(14,17,18)$. Thus the constant dose rate infusion was felt to provide plasma levels well in excess of those needed for radiosensitization. The dose was increased in $150 \mathrm{mg} / \mathrm{m}^{2} /$ week increments in successive cohorts of three patients until the DLT was observed. On days when gemcitabine was given, radiotherapy was delivered 4 hours after initiation of the drug infusion. This was based on preclinical data suggesting radiosensitization could occur as early as 4 hours after treatment, and lasting up to 2 days (17).

Radiotherapy was administered to the chest wall and regional nodes using techniques previously described (19). All patients had a pretreatment computed tomography (CT) scan with contrast to assess the extent of chest wall and regional nodal involvement. For patients with chest wall disease with or without supraclavicular disease, both chest wall and supraclavicular fossa regions were irradiated. If the axillary and/or internal mammary nodes were clinically involved, these nodal sites were included in the treatment volume. The entire chest wall was irradiated to 50 Gy utilizing opposed lateral tangential fields; 6 to 10 MV photon beams were used with either $0.5 \mathrm{~cm}$ of bolus every day or $1 \mathrm{~cm}$ of bolus every other day. For patients with no palpable supraclavicular disease, the supraclavicular fossa received 46 to $50 \mathrm{~Gy}$ to a depth of $3 \mathrm{~cm}$. For patients with supraclavicular involvement, a minimum dose of 50 Gy and maximum dose of 60 Gy was prescribed. Additional boosts of 10 to 20 Gy were administered to the chest wall to sites of gross disease using 9 to $12 \mathrm{MeV}$ electron 
beams, with the final dose dependent upon tumor bulk (20).

Toxicity grading was assessed using the Southwest Oncology Group (SWOG) Toxicity Criteria. DLT was defined as any grade 4 hematologic toxicity, grade 3 nonhematologic toxicity (except for alopecia, nausea, vomiting, or desquamation secondary to radiotherapy), or grade 3 dermatitis or desquamation appearing within the first 3 weeks of radiation. On-treatment visits with the treating physician as well as physical examinations and laboratory studies, including complete blood count, biochemistry panel with liver function tests, and urinalysis, were performed on a weekly basis for monitoring of toxicity during therapy.

Dose escalation of gemcitabine was performed after three patients treated at the same dose level were observed for a minimum of 4 weeks after completion of their treatments. If a DLT was encountered in one of three patients, then three additional patients were treated at the same dose; if only one of six patients experienced a DLT, then the subsequent group was treated at the next higher dose level. If the DLT was encountered in more than one of three to six patients at any dose level, the MTD would be declared as having been exceeded and dose escalation was terminated. The MTD was therefore defined as the dose level below which more than one of three to six patients developed a DLT.

If a DLT occurred, a break in the scheduled gemcitabine treatment was initiated until the toxicity had declined to grade 2 or less. Gemcitabine was then resumed at the next lower dose level. If a DLT occurred following a dose reduction, the patient was removed from the study. A dose reduction was initiated for any grade 2 hematologic toxicity or grade 3 dermatitis appearing after the first 3 weeks of radiation that was refractory to supportive measures. Withholding a dose was required for any grade 3 hematologic toxicity. Once a dose reduction or withholding occurred, the next dose was resumed at the full level. Radiation treatments continued without interruption except for a grade 3 skin desquamation not responsive to supportive measures.

Patients were seen in follow-up at 1 month after completion of their treatments. Clinical assessment of toxicity consisting of history, physical examination, and laboratory studies was performed. Patients were then followed every 2 months to assess tumor response and toxicity. Response grading was as follows: complete response (CR), no evidence of tumor; partial response (PR), at least a $50 \%$ decrease in tumor size based on physical examination and radiologic studies; stable disease, no change in tumor size; and progression, an increase of at least $25 \%$ in the size of measured lesions or the appearance of new lesions.

In addition, two historical cohorts of patients-those with unresectable chest wall failures treated with radiotherapy alone and those with resectable chest wall lesions treated with excision and postoperative radiotherapywere identified retrospectively from our institutional breast cancer registry by applying the same exclusion criteria used in our prospective phase I study. More specifically, the search criteria included any pre- or postmenopausal women with a history of breast cancer treated with mastectomy and/or systemic therapy without postmastectomy irradiation who underwent salvage radiotherapy with or without surgical excision for a chest wall recurrence. Kruskal-Wallis and Fisher exact chi-square tests were utilized to discern any differences in the baseline clinical characteristics among the three groups of patients. Crude rates of local-regional control for each treatment group were ascertained. Actuarial local-regional control curves were then generated utilizing the Kaplan-Meier technique, and overall differences among the three groups were further evaluated with a log-rank test to compare treatment outcome.

\section{RESULTS}

\section{Patient Characteristics}

Only six patients were required to reach the DLT, as discussed below. Three patients were treated at each dose level of 300 and $450 \mathrm{mg} / \mathrm{m}^{2} /$ week of gemcitabine. All patients were assessable for treatment-related toxicity and tumor control. Two groups of historical controls consisting of a total of 32 patients were identified from our computerized institutional breast cancer registry. Control group 1 consisted of eight patients with unresectable chest wall recurrences treated with radiotherapy alone; control group 2 consisted of 24 patients with resectable chest wall recurrences treated with excision followed by radiotherapy. Of note, none of these patients had any missing data that was required for the comparative analysis.

Clinical characteristics for each group are shown in Table 1 . The protocol and historical control 1 groups were similar except for treatment with a greater number of different chemotherapy regimens and less hormonal therapy, larger recurrent tumor burden, and a higher incidence of synchronous distant metastasis at the time of recurrent disease diagnosis among the protocol patients. Patients in historical control group 2 were more likely to have better Karnofsky Performance Status (KPS), present with earlier stages of disease prior to mastectomy, and less likely to 
Table 1. Comparison of Clinical Characteristics by Study Group

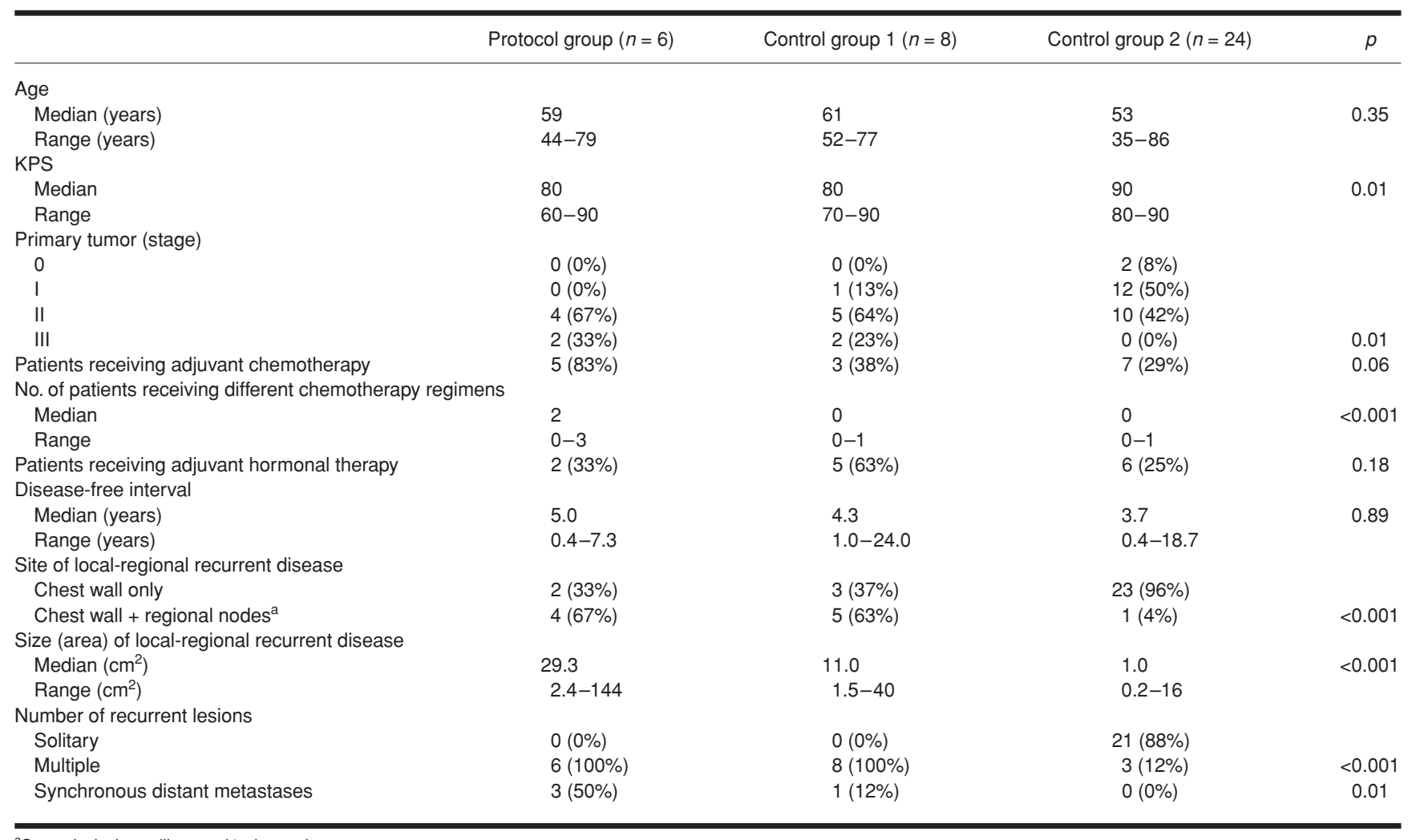

aSupraclavicular, axillary, and/or internal mammary.

have received chemotherapy in the past when compared to the other two groups. These patients were also more likely to have less tumor burden as manifested by smaller, solitary recurrences limited to the chest wall without synchronous distant metastases. Overall the protocol patients had the most aggressive chest wall failures, as evidenced by extensive local tumor burden and a higher percentage of synchronous distant metastases prior to initiation of gemcitabine and radiotherapy when compared with either of the two historical cohorts.

Median follow-up for the protocol, historical control 1 , and historical control 2 patients was 2 years (range $0.1-3.1$ years), 4 years (range $0.1-6.9$ years), and 6 years (range 1.0-13.5 years), respectively.

\section{Toxicity}

Dose-limiting toxicities occurred at the second planned dose of $450 \mathrm{mg} / \mathrm{m}^{2} /$ week in two of three patients. One patient experienced grade 4 thrombocytopenia as well as grade 3 neutropenia, while the other patient sustained a grade 4 neutropenia. Both patients were eventually removed from the study. The first patient experienced further neutropenia despite reduction and withholding of subsequent doses, and the latter patient experienced persistent grade 3 neutropenia as well a grade 3 radiationinduced skin desquamation refractory to conservative measures. The remaining patient did not incur a DLT and was able to receive six cycles of gemcitabine, with one dose reduction and one dose held due to hematologic toxicity. All three patients were able to complete their scheduled total radiotherapy doses of $70 \mathrm{~Gy}$, except for one patient who experienced significant grade 3 skin dermatitis near the end of her treatments and discontinued radiotherapy at $68 \mathrm{~Gy}$.

At the prior dose level of $300 \mathrm{mg} / \mathrm{m}^{2} /$ week, no DLT was encountered. All three patients were able to complete the planned gemcitabine cycles (two received six cycles and one received five cycles). Two patients each required one dose to be reduced to $200 \mathrm{mg} / \mathrm{m}^{2} /$ week and one dose to be held for hematologic toxicity according to the protocol guidelines. The remaining patient, who had the largest area of local-regional recurrence $\left(144 \mathrm{~cm}^{2}\right)$, experienced both hematologic and radiation-related skin toxicities, complicated by a transient idiosyncratic drug rash that necessitated four of her doses be reduced. She was, however, able to receive a total of five cycles of gemcitabine 
Table 2. Maximal Acute and Late Toxicities at $\mathbf{3 0 0}$ and $450 \mathrm{mg} / \mathrm{m}^{2} /$ week of Gemcitabine with Concurrent Radiotherapy

\begin{tabular}{lccccccccc}
\hline & \multicolumn{3}{c}{$300 \mathrm{mg} / \mathrm{m}^{2} /$ week $(n=3)$} & & $450 \mathrm{mg} / \mathrm{m}^{2} /$ week $(n=3)$ \\
\cline { 2 - 7 } Grade & 1 & 2 & 3 & 4 & 1 & 2 & 3 & 4 \\
\hline Neutropenia & - & 1 & 2 & - & - & - & 2 & $1^{\mathrm{a}}$ \\
Thrombocytopenia & - & 1 & - & - & - & - & - & $1^{\mathrm{a}}$ \\
Anemia & 3 & - & - & - & 1 & 2 & - & - \\
Desquamation & - & - & 3 & - & - & - & 3 & - \\
Drug rash & - & 1 & - & - & - & - & - & - \\
Elevated AST & 1 & - & - & - & - & - & - & - \\
Elevated ALT & 1 & - & - & - & - & - & - & - \\
Nausea & 2 & - & - & - & 1 & - & - & - \\
Fatigue & 1 & 1 & - & - & - & 1 & - & - \\
\hline
\end{tabular}

${ }^{\mathrm{a} D o s e-l i m i t i n g ~ t o x i c i t y . ~}$

and complete her radiotherapy. All three patients completed the full dose of 50 Gy to the chest wall and supraclavicular regions. They also received boost doses of an additional $20 \mathrm{~Gy}$ to sites of gross disease, except for one patient who withdrew from the study 3 days prior to the end of her treatments due to progressing systemic disease and declining performance status.

The toxicity profiles at both dose levels are shown in Table 2. In general, myelosuppression and the expected grade 3 radiotherapy-induced skin desquamation were most commonly observed. Some experienced mild nausea and fatigue, but no mucositis, emesis, diarrhea, pneumonitis, hepatotoxicity, proteinuria, flu syndrome, fever, or alopecia was noted. One patient incurred an idiosyncratic drug rash that resolved without intervention, while another patient experienced mild transitory elevations in AST and ALT that subsequently normalized without intervention.

\section{Response}

All six patients had significant clinical responses by the end of their treatments; three achieved complete responses while three experienced partial responses. Within 1 month of follow-up, all six patients had achieved complete regression of their treated local-regional disease based on clinical examination.

Thus the crude rate of local-regional control was $100 \%(6 / 6)$ in protocol patients treated with gemcitabine and radiotherapy. These results compared favorably to the crude rates of $50 \%$ (4/8 patients) and $83 \%(20 / 24$ patients) observed in the historical cohorts with unresectable recurrences treated with radiotherapy alone and resectable failures treated with excision followed by radiotherapy, respectively. Figure 1 shows a bar chart of crude control rates for the three groups $(p=0.060)$.

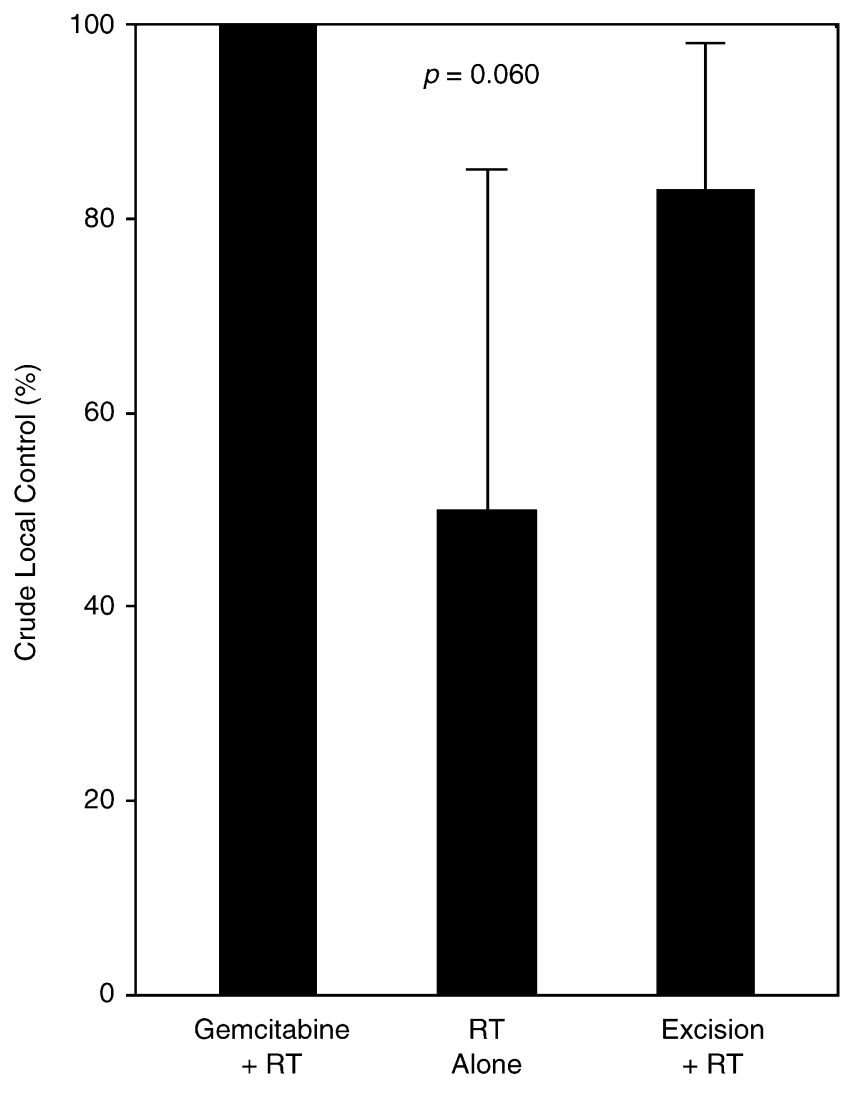

Figure 1. Bar chart of the crude rates of local-regional control using gemcitabine and radiotherapy, radiotherapy alone, and excision and radiotherapy for chest wall recurrences.

Actuarial rates of local-regional control were then assessed for the protocol patients (gemcitabine and radiotherapy) and compared to those of the two historical cohorts (radiotherapy alone; excision and radiotherapy) using the Kaplan-Meier method. Results are plotted in Figure 2. The radiotherapy alone group did worse with time, while the gemcitabine and radiotherapy group and excision and radiotherapy group fared similarly. The difference in local-regional control among the three groups was statistically significant $(p=0.007)$.

\section{DISCUSSION}

Isolated chest wall recurrences not amenable to gross resection pose a significant challenge for the treating radiation oncologist. These patients are at high risk of either persistent disease or recurrence following standard radiotherapy. Retrospective series have reported 5-year actuarial rates of local-regional recurrences as high as $76 \%$ with radiotherapy alone, despite optimal treatment planning and delivery $(4,6)$, leading to compromised quality of 


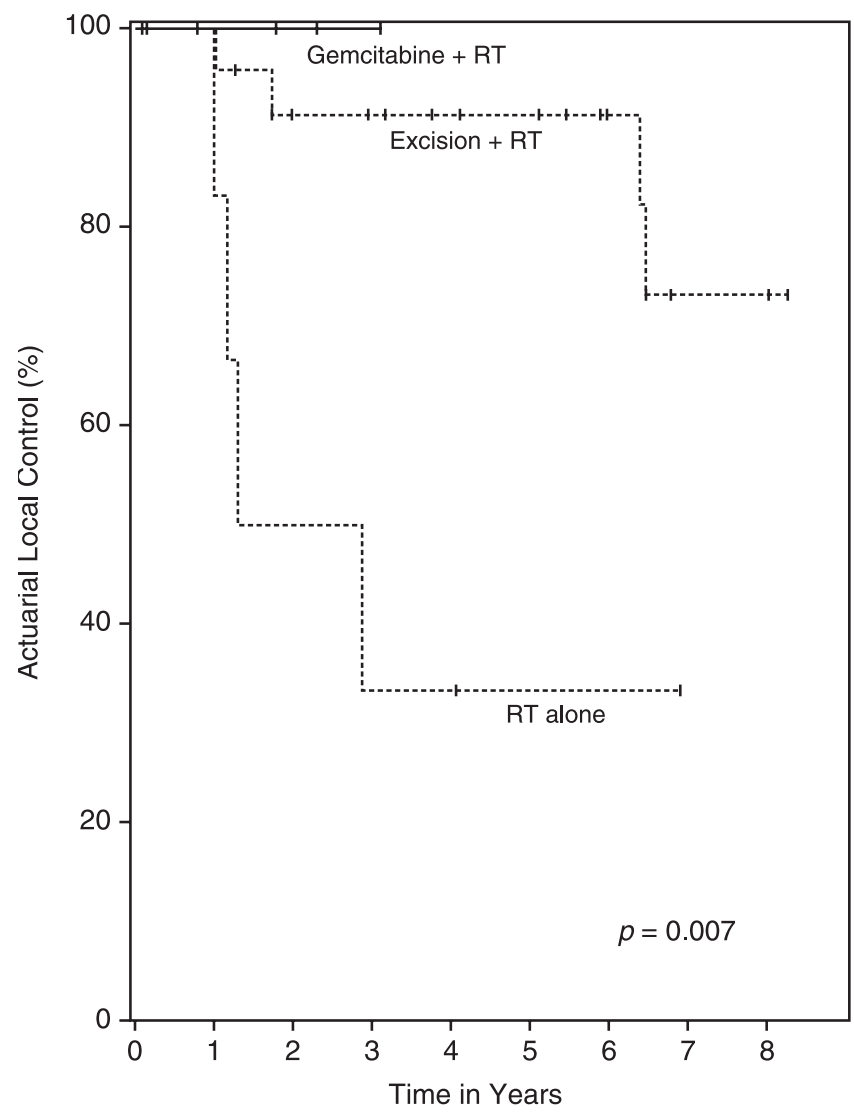

Figure 2. Actuarial estimates of local-regional control with gemcitabine and radiotherapy, excision and radiotherapy, and radiotherapy alone.

life (9). Thus effective local-regional treatment would be invaluable in this subset of recurrent breast cancer patients.

Although radiation dose escalation has been associated with increased tumor control, normal tissue tolerance limits delivery of the high doses required to sterilize bulky disease. Thus, due to the constraints of radiotherapy alone, the addition of concurrent chemotherapy to radiotherapy is a logical approach. The use of combined modality treatments is increasingly more common in the treatment of many solid tumors. Theoretical benefits of this approach include potential sterilization of systemic disease, as well as combined cytoreduction of local-regional tumor.

To our knowledge, this is the first clinical trial that has investigated the feasibility of delivering concurrent low-dose gemcitabine or any radiosensitizer with standard radiotherapy in breast cancer patients with unresectable chest wall recurrences. Gemcitabine is a drug with cytotoxic activity in breast cancer and radiation-sensitizing properties, both beneficial in the treatment of unresectable chest wall recurrences. Our study shows that for unresectable chest wall recurrences, the maximum tolerated dose of gemcitabine is $300 \mathrm{mg} / \mathrm{m}^{2} /$ week when given once a week with concurrent standard radiotherapy. Studies of gemcitabine given concomitantly with radiotherapy in pancreatic cancer have been reported with doses greater than $300 \mathrm{mg} / \mathrm{m}^{2} /$ week (21). However, this dose exceeded the MTD when given once a week with radiotherapy in patients with head and neck cancer (18). The low dose required in the current series probably reflects the extent of prior treatment with systemic therapies and limited bone marrow reserve. Nonetheless, there was excellent response at this dose, and the plasma concentrations exceeded the in vitro radiosensitizing concentration by at least 400-fold, suggesting that clinical benefit may also be achieved at lower doses for this group of patients.

Our study demonstrates that this novel regimen can be delivered safely for treatment of unresectable chest wall recurrences. The DLT, observed in two of the three patients at the dose level of $450 \mathrm{mg} / \mathrm{m}^{2} /$ week, was myelosuppression. Otherwise the most common toxicity encountered during treatment was the expected grade 3 skin dermatitis secondary to radiotherapy occurring by the end of therapy. All patients responded to supportive measures and did not require a break in their radiotherapy, except for one patient who received $450 \mathrm{mg} / \mathrm{m}^{2} /$ week of gemcitabine. Other than the hematologic and skinrelated toxicities, there were no other significant side effects except for a grade 2 idiosyncratic drug rash observed in one patient. All six patients were otherwise able to tolerate and complete their combined modality treatments as planned in accordance with the protocol guidelines.

A limited analysis of early treatment outcomes revealed that the protocol patients experienced excellent rates of local-regional control, as all six patients achieved complete response within 1 month of therapy. These results compared favorably to those of patients with unresectable failures treated traditionally with radiotherapy alone. When the protocol patients were compared to another group of historical patients with prognostically more favorable recurrences that were resectable and treated with postoperative radiotherapy, the local-regional control of the protocol patients was approximately equivalent if not better, although median follow-up was shorter and patient numbers were extremely limited in the protocol group. These limitations would suggest cautious interpretation of our results, but it should be noted that the favorable outcome observed in the present series occurred despite a greater documented tumor burden in the protocol patients when compared to the other two historical 
cohorts. Thus chest wall irradiation with concurrent gemcitabine, a drug which has both cytotoxic and radiationsensitizing properties, appears to have the potential to improve local-regional outcome, and formal study using a larger patient cohort in a phase II trial is warranted.

\section{CONCLUSION}

Concurrent gemcitabine at $300 \mathrm{mg} / \mathrm{m}^{2} /$ week and radiotherapy can be safely administered for unresectable chest wall recurrences. Toxicities were mainly limited to myelosuppression and expected, yet well-tolerated, radiotherapy-induced moist desquamation. Early results using this regimen suggest high rates of local-regional control in patients with unresectable disease comparable to rates achieved in historical controls treated with complete excision followed by comprehensive radiotherapy. This novel approach of combining gemcitabine with radiotherapy may offer better local control when compared to radiotherapy alone for unresectable recurrences. A phase II trial is currently under way to study the efficacy of this combined modality approach.

\section{Acknowledgments}

Presented at the 44th annual meeting of the American Society of Therapeutic Radiology and Oncology in New Orleans, Louisiana, October 6, 2002.

\section{REFERENCES}

1. Deck KB, Kern WH. Local recurrence of breast cancer. Arch Surg 1976;111:323-25.

2. Fisher B, Redmond C, Poisson R, et al. Eight-year results of a randomized clinical trial comparing total mastectomy and lumpectomy with or without radiation in the treatment of breast cancer. N Engl J Med 1989;320:822-28.

3. Crowe JP Jr, Gordon NH, Antunez AR, et al. Local-regional breast cancer recurrence following mastectomy. Arch Surg 1991;126:429-32.

4. Aberizk WJ, Silver B, Henderson IC, et al. The use of radiotherapy for treatment of isolated locoregional recurrence of breast carcinoma after mastectomy. Cancer 1986;58:1214-18.

5. Bedwinek JM, Lee J, Fineberg B, et al. Prognostic indicators in patients with isolated local-regional recurrence of breast cancer. Cancer 1981;47:2232-35.

6. Schwaibold F, Fowble BL, Solin LJ, et al. The results of radiation therapy for isolated local regional recurrence after mastectomy. Int $\mathrm{J}$ Radiat Oncol Biol Phys 1991;21:299-310.

7. Stadler B, Kogelnik HD. Local control and outcome of patients irradiated for isolated chest wall recurrences of breast cancer. Radiother Oncol 1987;8:105-11.

8. Halverson KJ, Perez CA, Kuske RR, et al. Isolated local-regional recurrence of breast cancer following mastectomy: radiotherapeutic management. Int J Radiat Oncol Biol Phys 1990;19:851-58.

9. Bedwinek JM, Fineberg B, Lee J, et al. Analysis of failures following local treatment of isolated local-regional recurrence of breast cancer. Int J Radiat Oncol Biol Phys 1981;7:581-85.

10. Lund B, Kristjansen PEG, Hansen HH. Clinical and preclinical activity of 2',2'-difluorodeoxycytidine (gemcitabine). Cancer Treat Rev 1993;19:45-55

11. Carmichael J, Possinger K, Phillip P, et al. Advanced breast cancer: a phase II trial with gemcitabine. J Clin Oncol 1995;13:273136.

12. Shewach DS, Hahn TM, Chang E, et al. Metabolism of $2^{\prime}, 2^{\prime}-$ difluoro-2'-deoxycytidine and radiation sensitization of human colon carcinoma cells. Cancer Res 1994;54:3218-23.

13. McGinn CJ, Shewach DS, Lawrence TS. Radiosensitizing nucleosides. J Natl Cancer Inst 1997;88:1193-203.

14. Robinson WR, Shewach DS. Radiosensitization by gemcitabine in p53 wild-type and mutant MCF-7 breast carcinoma cell lines. Clin Cancer Res 2001;7:2581-89.

15. Abbruzzese JL, Grunewald R, Weeks EA, et al. A phase I clinical, plasma, and cellular pharmacology study of gemcitabine. J Clin Oncol 1991;9:491-98.

16. Gruenwald R, Kantarjian H, Keating MJ, et al. Pharmacologically directed design of the dose rate and schedule of $2^{\prime}, 2^{\prime}$ difluorodeoxycitidine (gemcitabine) administration in leukemia. Cancer Res 1990;50:6823-26.

17. Lawrence TS, Eisbruch A, Shewach DS. Gemcitabine-mediated radiosensitization. Semin Oncol 1997;24(2 Suppl 7):S7-24-S7-28.

18. Eisbruch A, Shewach DS, Bradford CR, et al. Radiation concurrent with gemcitabine for locally advanced head and neck cancer: a phase I trial and intracellular drug incorporation study. J Clin Oncol 2001;19:792-99.

19. Pierce LJ, Strawderman MH, Douglas KR, et al. Conservative surgery and radiotherapy for early-stage breast cancer using a lung density correction: the University of Michigan experience. Int J Radiat Oncol Biol Phys 1997;39:921-28.

20. Bedwinek J. Natural history and management of isolated local-regional recurrence following mastectomy. Semin Radiat Oncol 1994;4:260-69.

21. McGinn CJ, Zalupski MM, Shureiqi I, et al. Phase I trial of radiation dose escalation with concurrent weekly full-dose gemcitabine in patients with advanced pancreatic cancer. J Clin Oncol2001;19:42028. 